\title{
Educación emocional en jóvenes con discapacidad intelectual: evaluación de una intervención en contexto universitario
}

\author{
Emotional education in young people with intellectual disabilities: \\ Assessment of an intervention in university context
}

\begin{abstract}
Resumen
El objetivo del presente estudio fue analizar el impacto de una intervención en educación emocional en un grupo de jóvenes con discapacidad intelectual. Los participantes fueron 16 jóvenes (7 mujeres) con discapacidad intelectual ligera o moderada de edades comprendidas entre los 18 y 28 años $(M=24 ; D T=3,04)$ integrantes del programa de formación para el empleo UNINCLUV de la Universitat de València. Se administraron el cuestionario de Inteligencia Emocional de Bar-On: versión para jóvenes (EQ-i:YV; Bar-On y Parker, 2000) y la Escala de Comportamiento Asertivo (CABS; Woods et al., 1978). El diseño del estudio fue longitudinal con pretest, intervención y postest, sin grupo control. No se observaron diferencias en las puntuaciones obtenidas por los participantes antes y después del tratamiento en las distintas dimensiones de inteligencia emocional evaluadas (intrapersonal, interpersonal, estrés, adaptabilidad, estado de ánimo). Sin embargo, sí se observó un mayor uso de estrategias asertivas y un menor uso de estrategias pasivas entre los participantes tras la intervención. Asimismo, los participantes manifestaron un elevado grado de satisfacción con el programa. Los resultados obtenidos indican la necesidad de continuar con la implementación de programas universitarios que potencien tanto la inclusión como las competencias socioemocionales de las personas con discapacidad intelectual, con el fin de mejorar la calidad de vida de este colectivo.
\end{abstract}

\section{Palabras clave}

Discapacidad intelectual, educación emocional, contexto universitario, inclusión.

\begin{abstract}
The goal of the present study was to analyze the impact of an intervention in emotional education in a group of young people with intellectual disabilities. The participants were 16 young people ( 7 women) with mild or moderate intellectual disability between the ages of 18 and $28(M=24 ; S D=3.04)$ who were taking part of the UNINCLUV employment training program at the University of Valencia. The Bar-On Emotional Intelligence questionnaire: youth version (EQ-i:YV; Baron and Parker, 2000) and the Assertive Behavior Scale (CABS; Woods et al., 1978) were administered. The study design was longitudinal with pretest, intervention, and posttest, without control group. No differences were observed in the scores obtained by the participants before and after treatment in the different dimensions of emotional intelligence (intrapersonal, interpersonal, stress, adaptability, mood). However, there was a greater use of assertive strategies and a lower use of passive strategies among the participants after the intervention. Likewise, the participants expressed a high degree of satisfaction with the program. The results indicate the need to continue with the design and implementation of university programs that enhance both the inclusion and the socio-emotional skills with intellectual disabilities, in order to improve the quality of life of this group.
\end{abstract}

\section{Keywords}

Intellectual disabilities, emotional education, university context, inclusion.

\author{
Beatriz Lucas-Molina \\ <beatriz.lucas@uv.es> \\ Universitat de València. España
}

\section{Vicenta Ávila Clemente <vicenta.avila@uv.es>}

Universitat de València. España

Alicia Pérez de Albéniz
<alicia.perez@unirioja.es>
Universidad de la Rioja. España

Eduardo Fonseca-Pedrero <eduardo.fonseca@unirioja.es>

Universidad de la Rioja. España

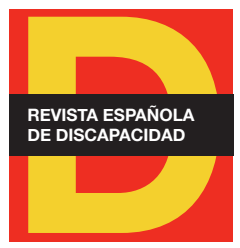

\begin{abstract}
Para citar:
Lucas-Molina, B. et al. (2020). "Educación emocional en jóvenes con discapacidad intelectual: evaluación de una intervención en contexto universitario". Revista Española de Discapacidad, 8(2), pp. 151-162.
\end{abstract}

Doi: <https://doi.org/10.5569/23405104.08.02.07>

Fecha de recepción: 16-06-2020 Fecha de aceptación: 30-11-2020

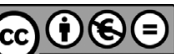




\section{Introducción}

Las universidades desempeñan un papel fundamental en la inclusión social de las personas con discapacidad (Izuzquiza, 2012). En este sentido, en los últimos años se han venido realizando esfuerzos desde el contexto universitario para asegurar los principios de igualdad de oportunidades y de integración a las personas con discapacidades físicas y sensoriales (Palazón et al., 2011). A pesar de esto, recientes estudios señalan que los estudiantes universitarios con discapacidad son una excepción, suponiendo solo un 1,7\% del estudiantado universitario (Fundación Universia, 2017). Si bien no se dispone de cifras concretas, parece que este panorama es aún más desolador para los jóvenes con discapacidad intelectual, cuya presencia en los espacios de educación superior es prácticamente testimonial (Cerrillo et al., 2013). Resulta pues evidente que el contexto universitario necesita seguir adaptándose para responder a las demandas formativas de este colectivo y promover con ello su inclusión real.

Desde hace dos años se viene desarrollando en la Universitat de València (en adelante UV) el programa universitario de formación para el empleo, UNINCLUV, dirigido a jóvenes con discapacidad intelectual. Esta iniciativa se enmarca a su vez en el programa Unidiversidad, impulsado por la Fundación ONCE con el apoyo del Fondo Social Europeo, cuyo objetivo principal es implicar a las universidades en la formación e inserción laboral de jóvenes con discapacidad intelectual para que puedan convertirse en adultos independientes y contribuir plenamente a la sociedad. Por consiguiente, el fin último del programa Unidiversidad es crear entornos universitarios y sociedades verdaderamente inclusivos. En la actualidad, son 23 las universidades españolas que han recibido financiación de la Fundación ONCE para llevar a cabo este tipo de programas formativos'.

Las evaluaciones de las experiencias nacionales e internacionales desarrolladas en esta línea ponen de manifiesto sus efectos positivos tanto en la empleabilidad (Miller et al., 2016; Moore y Schelling, 2015; Ross et al., 2013), como en distintos indicadores de bienestar emocional y relacional de las personas con discapacidad intelectual participantes (Fuller et al., 2006; Prohn et al., 2019; Ross et al., 2013). Cabe señalar que estas iniciativas inclusivas suponen también una fuente de enriquecimiento para toda la comunidad universitaria y para la sociedad en su conjunto (Cerrillo et al., 2013; Miller et al., 2016; Prohn et al., 2019; Ryan y Struths, 2004).

Como es bien sabido, la discapacidad intelectual se caracteriza por limitaciones significativas tanto en el funcionamiento intelectual como en la conducta adaptativa, entendida esta última como el conjunto de habilidades conceptuales, sociales y prácticas aprendidas por las personas para funcionar en su vida diaria (Verdugo et al., 2011). En consecuencia, para poder mejorar el funcionamiento y la calidad de vida de las personas con discapacidad intelectual, es necesario incidir en estas habilidades adaptativas (Navas et al., 2010). Es por ello que, si bien dentro del ámbito universitario español existe una gran heterogeneidad en los planes de estudios ofertados por los distintos programas de formación para personas con discapacidad intelectual (Cerrillo et al., 2013), la mayoría de estos suelen trabajar de una u otra forma los tres dominios (conceptual, social y práctico) de la conducta adaptativa. En particular, dentro del dominio social, las competencias socioemocionales suelen incluirse como competencias básicas a desarrollar.

1. Información obtenida de https://www.fundaciononce.es/es/pagina/iii-convocatoria-de-ayudas-para-programas-universitarios-de-formacion-para-el-empleo. 
En el caso concreto de la UV, el plan de estudios del programa UNINCLUV consta de 33,7 créditos ECTS organizados en 6 módulos distintos ${ }^{2}$ y desarrollados a lo largo de seis meses (Noviembre-Mayo) en la Facultad de Psicología. Dentro del "Módulo 2: Formación en comunicación, habilidades sociales, emocionales, entrenamiento cognitivo" se incluye una asignatura de carácter obligatorio denominada "Gestión emocional y habilidades sociales" que tiene una duración de 1,6 créditos ECTS. Es en esta asignatura donde se enmarca la presente investigación.

Diversos estudios han puesto de manifiesto que la educación en competencias socioemocionales, entendidas como el conjunto de conocimientos, capacidades, habilidades y actitudes necesarias para comprender, expresar y regular de forma apropiada los fenómenos emocionales para el establecimiento de relaciones inter e intrapersonales adecuadas (Bisquerra et al., 2015), resultan claves para conseguir el bienestar psicológico y la adaptación del individuo (Bisquerra et al., 2015; Fernández-Berrocal et al., 2017). Esto es especialmente relevante para las personas con discapacidad intelectual ya que suelen presentar mayores dificultades en el manejo de su mundo emocional y sus relaciones sociales (La Malfa et al., 2009; Rey et al., 2013). Además, al igual que en la población general, se ha observado una estrecha relación entre competencia socioemocional y calidad de vida subjetiva en las personas con discapacidad intelectual (Rey et al., 2013). Es por ello por lo que, a la hora de promover la inclusión laboral de este colectivo, resulta imprescindible fomentar en ellos y ellas las habilidades sociales y emocionales necesarias para poder relacionarse con éxito en los diferentes entornos profesionales en los que se encuentren, así como para el adecuado trabajo en equipo.

En el campo de la intervención en educación emocional en contextos formales no universitarios destacan los programas basados en el modelo propuesto por el grupo de investigación GROP (Bisquerra, 2000; 2002; Bisquerra y Pérez, 2007). Son diversos los estudios que han puesto de manifiesto la efectividad de estos programas para mejorar las habilidades emocionales y sociales en niños y adolescentes (Filella et al., 2014; Muñoz de Morales y Bisquerra, 2013). Dentro del colectivo de la discapacidad intelectual, son aún escasos los trabajos que examinen la eficacia de las intervenciones en educación emocional. Entre las excepciones se encuentra el trabajo de González et al. (2019), que evaluaron una intervención en población reclusa con discapacidad intelectual, encontrando un mayor autodominio y manejo de sus sentimientos y emociones, así como un aumento de la empatía y de las conductas asertivas. Esta intervención estaba basada también en el modelo de competencias socioemocionales del grupo GROP (Bisquerra et al., 2015).

En línea con lo anterior, hasta donde conocemos, no existen estudios que analicen el impacto de este tipo de intervenciones dentro de los programas formativos universitarios españoles. Por lo tanto, parece razonable diseñar, implementar y evaluar intervenciones en educación emocional en el entorno universitario con la finalidad de dotar a los jóvenes con discapacidad intelectual de recursos y estrategias socioemocionales para afrontar los retos que se les presenten en la vida cotidiana, especialmente en el ámbito laboral, y con ello mejorar su calidad de vida. En concreto, dado que las intervenciones fundamentadas en el modelo de Bisquerra (Bisquerra, 2000; 2002; Bisquerra y Pérez, 2007) han ofrecido resultados positivos tanto entre la población general como con discapacidad intelectual, parece sensato adaptarlas y aplicarlas en este colectivo en el entorno universitario.

2. Para más información sobre el plan de estudios se puede consultar http://esdeveniments.uv.es/40608/section/21468/unincluv-2o-edicion_programa-de-formacion-para-el-empleo-de-jovenes-con-discapacidad-intelectual.html. 
Dentro de este contexto de investigación, la finalidad del presente estudio es: a) implementar una intervención en educación emocional dentro de un programa universitario para jóvenes con discapacidad intelectual; b) analizar los efectos de la intervención a través de indicadores de inteligencia emocional y conducta social percibida, y c) examinar la satisfacción con la intervención de los usuarios. Se espera que los participantes refieran mejorías en inteligencia emocional y asertividad tras la intervención, así como una elevada satisfacción con la intervención.

\section{Método}

\subsection{Participantes}

Los participantes fueron 16 alumnos (7 chicas) del programa UNINCLUV, con edades comprendidas entre los 18 y 28 años $(M=24 ; D T=3,01)$, con diagnóstico reconocido de discapacidad intelectual ligera o moderada.

El diseño de la investigación es longitudinal con pretest, tratamiento y postest, sin grupo control. No se pudo aleatorizar la intervención ni obtener un grupo control dadas las características del contexto en el que se realizó la intervención, así como de los propios participantes. En este sentido, la principal dificultad de este estudio será diferenciar los posibles efectos de la intervención de aquellos debidos a otros factores no controlados.

\subsection{Instrumentos}

- Cuestionario de inteligencia emocional (Emotional Quotient inventory: Young Version, EQ-i:YV; Bar-On y Parker, 2000). El EQ-i:YV es un autoinforme diseñado para medir la inteligencia emocional en niños y adolescentes de 7 a 18 años. Se consideró la adecuación de su uso dada la edad equivalente de vocabulario en PEABODY (Dunn et al., 2007). Consta de 60 ítems de respuesta tipo Likert de cuatro puntos ( 1 = Raramente me pasa o No es cierto; 4 = Frecuentemente me pasa o Es cierto). Valora cinco dimensiones: Competencias Intrapersonales (6 ítems), Competencias Interpersonales (12 ítems), Manejo del Estrés (12 ítems), Adaptabilidad (10 ítems) y Estado de Ánimo (14 ítems). Asimismo, incluye una sexta dimensión, Impresión Positiva, compuesta por 6 ítems que tiene como objetivo medir el grado en que los sujetos responden al azar o distorsionan sus respuestas en función de la deseabilidad social. Para el presente estudio se utilizó la versión del EQ-i:YV validada en población española (Ferrándiz et al., 2012), cuyas puntuaciones han mostrado una adecuada fiabilidad (entre 0,63 y 0,80) en muestras de estudiantes entre 6 y 18 años.

- Escala de comportamiento asertivo para niños (Children's Assertive Behavior Scale, CABS; Woods et al. , 1978). Este autoinforme proporciona información sobre el comportamiento asertivo y no asertivo en niños y adolescentes y permite conocer el estilo de relación (asertivo, pasivo o agresivo). La escala tiene 27 ítems con cinco alternativas de respuesta que oscilan en un continuo de pasividad a agresividad: pasiva, parcialmente pasiva, asertiva, parcialmente agresiva, agresiva. El participante tiene que escoger aquella que más 
identifique su comportamiento social habitual. La respuesta pasiva recibe la mínima puntuación (1) y la agresiva la máxima (5). A partir de este cuestionario pueden obtenerse tres tipos de puntuaciones: las puntuaciones pasivas (número de veces que el participante es principalmente pasivo), las puntuaciones agresivas (número de veces que el participante es principalmente agresivo) y las puntuaciones asertivas (número de veces que el participante es principalmente asertivo). El contenido de algunos de los ítems fue modificado para adaptarse a las edades de los participantes. La versión en español ha mostrado niveles adecuados de fiabilidad (entre 0,65 y 0,72 ) entre estudiantes de primaria (de la Peña et al., 2003).

- Cuestionario de satisfacción. Para valorar el grado de satisfacción general de los participantes con la intervención se desarrolló un breve cuestionario ad hoc compuesto por cuatro ítems. Dos de los ítems: En general, ¿te ha gustado esta asignatura? y ¿Cómo ha sido tu relación con el profesor o profesora de esta asignatura?, tenían un formato de respuesta tipo Likert de 4 opciones de respuesta ( 1 = No me ha gustado nada; 4 = Me ha gustado mucho) y 5 ( 1 = Muy mala; $5=$ Muy buena) puntos, respectivamente. Las otras dos preguntas; ¿Qué es lo que más te ha gustado de esta asignatura?, ¿Por qué? y ¿Qué es lo que menos te ha gustado?, ¿Por qué?, eran de tipo abierto y tenían como objetivo detectar aquellos aspectos positivos y negativos más relevantes de la intervención recibida.

\subsection{Procedimiento}

La intervención en educación emocional se desarrolló dentro del curso "Gestión emocional y habilidades sociales" perteneciente al "Módulo 2. Formación en comunicación, habilidades sociales, emocionales y entrenamiento cognitivo" del programa UNINCLUV de la UV. Tuvo una duración total de 16 horas, distribuidas en cuatro sesiones de cuatro horas de duración a lo largo de dos semanas.

La intervención implementada se basó en el programa de Inteligencia Emocional para educación secundaria desarrollado por la Diputación de Guipúzcoa (Aierdi et al., 2008), cuya efectividad ha sido puesta de manifiesto en estudios previos (Muñoz de Morales y Bisquerra, 2013). Este programa está fundamentado a su vez en el modelo de competencias emocionales de Bisquerra $(2000,2002)$. En este modelo se diferencian cinco grandes competencias (Bisquerra y Pérez, 2007): la conciencia emocional, la regulación emocional, la autonomía emocional, las habilidades sociales y las habilidades de vida y bienestar. Dada la breve duración de la intervención y las características de los participantes, se optó por desarrollar únicamente tres competencias: la conciencia emocional, la regulación emocional y las habilidades sociales. Asimismo, se adaptaron muchas de las actividades a las características de los participantes para que resultasen significativas para ellos. En la Tabla 1 se presenta, de forma abreviada, las sesiones llevadas a cabo en el programa de intervención, los objetivos, el contenido y las actividades realizadas. 


\begin{tabular}{|c|c|c|c|}
\hline Sesión & Objetivos & Contenidos & Actividades \\
\hline $\begin{array}{l}\text { 1. Conciencia } \\
\text { Emocional }\end{array}$ & $\begin{array}{l}\text { - Evaluación inicial de los } \\
\text { participantes. } \\
\text { - Conocer las emociones } \\
\text { básicas y cómo estas nos } \\
\text { ayudan. } \\
\text { - Expresar las emociones de } \\
\text { distintas maneras. }\end{array}$ & $\begin{array}{l}\text { - Introducción, presentación y } \\
\text { evaluación. } \\
\text { - Reconocimiento de las } \\
\text { emociones básicas a través } \\
\text { de imágenes y documentos. } \\
\text { audiovisuales. } \\
\text { - Expresión de las emociones } \\
\text { de diferentes maneras. }\end{array}$ & $\begin{array}{l}\text { - Instrumentos de } \\
\text { - } \text { Conaluación. } \\
\text { - Emozco las emociones. } \\
\text { - de mis emociones. } \\
\text { - Cómo me siento cuándo.... } \\
\text { - Adivina la emoción. }\end{array}$ \\
\hline $\begin{array}{l}\text { 2. Regulación } \\
\text { Emocional }\end{array}$ & $\begin{array}{l}\text { - Saber distinguir las } \\
\text { emociones, la conducta y el } \\
\text { pensamiento. } \\
\text { - Aprender a regular } \\
\text { eficazmente las emociones. }\end{array}$ & $\begin{array}{l}\text { - Conocimiento de la } \\
\text { interacción entre emoción, } \\
\text { pensamiento y conducta. } \\
\text { - Utilización de estrategias } \\
\text { que favorezcan la regulación } \\
\text { emocional. }\end{array}$ & $\begin{array}{l}\text { - } \text { Actuamos como } \\
\text { pensamos. } \\
\text { - Yo regulo mis emociones. } \\
\text { - Pues...voy a decírtelo. } \\
\text { - } \quad \text { Vamos a relajarnos! }\end{array}$ \\
\hline $\begin{array}{l}\text { 3. Habilidades } \\
\text { Sociales }\end{array}$ & $\begin{array}{l}\text { - Aprender a mostrar los } \\
\text { sentimientos de forma } \\
\text { asertiva. } \\
\text { - Adquirir habilidades para la } \\
\text { interacción asertiva. }\end{array}$ & $\begin{array}{l}\text { - Conocimiento de los estilos } \\
\text { de interacción: pasivo, } \\
\text { asertivo y agresivo. } \\
\text { - Habilidades de interacción } \\
\text { asertivas. }\end{array}$ & $\begin{array}{l}\text { - De vacaciones. } \\
\text { - Yo lo mío, tú lo tuyo, cada } \\
\text { uno a lo suyo. }\end{array}$ \\
\hline $\begin{array}{l}\text { 4. Habilidades } \\
\text { Sociales }\end{array}$ & $\begin{array}{l}\text { - Adquirir habilidades para } \\
\text { hacer frente de forma } \\
\text { asertiva a los conflictos } \\
\text { - Adquirir habilidades para } \\
\text { resistir a la presión grupal. }\end{array}$ & $\begin{array}{l}\text { - Habilidades de resolución de } \\
\text { conflictos asertivas. } \\
\text { - Técnicas para resistir a la } \\
\text { presión de grupo. }\end{array}$ & $\begin{array}{l}\text { - Ni pensarlo, no pienso } \\
\text { hacerlo. } \\
\text { - Tú ganas, yo también } \\
\text { gano. }\end{array}$ \\
\hline
\end{tabular}

Fuente: elaboración propia.

La metodología utilizada fue fundamentalmente de carácter práctico. El alumnado trabajó de forma cooperativa en el aula en pequeños grupos heterogéneos (máximo 4 personas) según su nivel de competencia en las distintas temáticas tratadas puesto de manifiesto en la evaluación inicial a través del EQ-i:YV y el CABS. Para ello, se establecieron cuatro niveles (bajo, medio-bajo, medio-alto y alto) en las dimensiones Competencias Interpersonales y Manejo del Estrés del EQ-i:YV y en el número de respuestas pasivas, agresivas y asertivas obtenidas por los participantes en el CABS. De este modo, se intentó que todos los grupos estuviesen compuestos por participantes de cada uno de los cuatro niveles de competencia establecidos.

Las actividades fueron adaptadas y diseñadas para que el alumnado pusiese en práctica a través de la dramatización de situaciones ficticias y significativas, las distintas habilidades emocionales y sociales desarrolladas en el curso. Las estrategias que se utilizaron con mayor frecuencia fueron el modelado, la práctica guiada y el refuerzo positivo. Mediante estas estrategias se pretendía fomentar no solo el aprendizaje del alumnado, sino también su sentimiento de autoeficacia y autonomía. En la Figura 1 se presenta un ejemplo de la actividad "Yo lo mío, tú lo tuyo, cada uno a lo suyo" correspondiente a la Sesión 3 del programa. En esta actividad, después de tratar las desventajas de los estilos de interacción pasivo y agresivo, así como presentar y ejemplificar de forma sencilla el estilo asertivo, se presentaban cuatro situaciones distintas de la vida cotidiana (trabajo, amistad, relación de pareja, relación familiar) basadas en experiencias personales aportadas por el propio alumnado. A partir de la situación entregada, el alumnado tenía que inventar un guion a modo de diálogo siguiendo el esquema trabajado sobre el estilo asertivo (explicar la conducta que molesta, expresar el sentimiento que genera, informar de lo que se espera de la persona, expresar lo que 
puede ocurrir si la persona cambia o no cambia su conducta), para después dramatizarlo. El objetivo de esta actividad era que el alumnado practicase y se apropiase del estilo de interacción asertivo.

Figura 1. Ejemplo actividad "Yo lo mío, tú lo tuyo, cada uno a lo suyo", Sesión 3

\section{ACTIVIDAD 3.2. YO LO MÍO, TÚ LO TUYO, CADA UNO/A LO SUYO}

A menudo, las personas de nuestro entorno no se dan cuenta de que están infringiendo nuestros derechos. Cuando hay algo que nos molesta o desagrada es muy importante decir lo que pensamos y pedir al otro que cambie esa conducta inadecuada. Para ello hemos visto una estrategia muy útil para poder decir aquello que nos molesta de manera positiva. En esta actividad vamos a aprovechar para practicar esa estrategia.

1. Leed la siguiente historia en grupo.

Hace aproximadamente un mes un compañero de trabajo te pidió un cambio de turno (que hicieses tú el de noche y él el de día) porque ese día era el aniversario de su mujer y quería ir a celebrarlo con una cena. A cambio te comentó que te devolvería el favor y te cubriría una noche de la siguiente semana. Cuando llega el día acordado, dice que no puede, que le es imposible por un tema familiar. Pasan los días y las semanas y parece que evita hablar del tema. ¿Cómo podrías abordar esta situación?

2. Plantead un diálogo para resolver la situación de forma asertiva. Ayudaos del 'truco' que hemos visto en clase, inunca falla!:

Cuando...

Me siento...

Me gustaría...

Si no es así/si es así....

3. ¡A representar la situación!

Fuente: elaboración propia.

Los cuestionarios fueron cumplimentados de forma colectiva por los participantes, previo consentimiento informado de las familias o del propio participante. Durante la administración se prestó todo el apoyo necesario teniendo en cuenta las características del colectivo. En todo momento se aseguró el tratamiento anónimo y confidencial de la información obtenida. La evaluación pretest tuvo lugar en la primera sesión de la intervención, mientras que la postest (junto con el cuestionario de satisfacción) se llevó a cabo a los 10 días de haber realizado la última sesión. 


\subsection{Análisis de los datos}

En primer lugar, se calcularon los estadísticos descriptivos para las subescalas de los diferentes instrumentos de medida utilizados. En segundo lugar, se compararon las puntuaciones medias en función del tiempo (pretest y postest) para ver si existían diferencias estadísticamente significativas. Para ello se llevó a cabo un Análisis Multivariado de la Varianza (MANOVA) de medidas repetidas, tomando el tiempo como factor intrasujeto y como variables dependientes las subescalas. Se utilizó el valor Lambda de Wilks para observar si existían diferencias significativas entre todas las variables dependientes tomadas en conjunto. Como índice de tamaño del efecto se empleó el estadístico eta cuadrado parcial ( $\eta^{2}$ parcial). Cuando $\eta^{2}$ parcial > 0,14 el efecto es grande en magnitud, y cuando $\eta^{2}$ parcial $<0,06$ el tamaño del efecto es medio. Finalmente, se calcularon los estadísticos descriptivos para la medida de satisfacción con el programa. Para el análisis de los datos se utilizó el programa estadístico SPSS 21.0 (IBM, 2013).

\section{Resultados}

\section{- Efectos de la intervención en la inteligencia emocional}

En la Tabla 2 se presentan las medias y las desviaciones típicas en las cinco escalas del EQ-i:YV antes y después de la intervención. Tal y como puede observarse en dicha tabla, las puntuaciones son prácticamente idénticas en ambos momentos temporales. En este sentido, el valor de $\lambda$ de Wilks $(\lambda$ Wilks $=0,649, F=$ $0,975, p=.482, \eta^{2}$ parcial $=0,351$ ) reveló que el efecto del factor tiempo no era estadísticamente significativo. Por lo tanto, no se produjeron cambios en las distintas dimensiones de inteligencia emocional evaluadas en el EQ-i:YV como efecto de la intervención.

\begin{tabular}{l}
\hline Tabla 2. Medias y desviaciones típicas en las distintas dimensiones del EQ-i:YV en ambos momentos temporales (N=14) \\
\begin{tabular}{|l|c|c|c|c|c|}
\hline & $\begin{array}{c}\text { Intrapersonal } \\
(\boldsymbol{M}, \boldsymbol{D} \boldsymbol{t})\end{array}$ & $\begin{array}{c}\text { Interpersonal } \\
(\boldsymbol{M}, \boldsymbol{D} \boldsymbol{t})\end{array}$ & $\begin{array}{c}\text { Estrés } \\
(\boldsymbol{M}, \boldsymbol{D} \boldsymbol{t})\end{array}$ & $\begin{array}{c}\text { Adaptabilidad } \\
(\boldsymbol{M}, \boldsymbol{D} \boldsymbol{t})\end{array}$ & $\begin{array}{c}\text { Estado de ánimo } \\
(\boldsymbol{M}, \boldsymbol{D} \boldsymbol{t})\end{array}$ \\
\hline Pre & $16,57(2,87)$ & $40,00(5,14)$ & $37,07(4,81)$ & $28,57(6,45)$ & $47,43(6,81)$ \\
\hline Post & $15,67(3,40)$ & $39,60(5,62)$ & $35,16(7,87)$ & $28,16(7,23)$ & $45,69(8,09)$ \\
\hline
\end{tabular}
\end{tabular}

Nota: el número de ítems y por tanto el rango de respuestas para cada una de las escalas no es el mismo. Intrapersonal (6 ítems, rango: 0-24), Interpersonal (12 ítems, rango: 0-48), Estrés (12 ítems, rango: 0-48), Adaptabilidad (10 ítems, rango: 0-40) y Estado de ánimo (14 ítems, rango: 0-56).

Fuente: elaboración propia.

\section{- Efectos de la intervención en el comportamiento social}

Como puede observarse en la Tabla 3, tanto antes como después de la intervención, los participantes emplearon con mayor frecuencia las respuestas asertivas, seguidas de las pasivas y en último lugar de las agresivas. El valor de $\lambda$ de Wilks $\left(\lambda\right.$ Wilks $=0,523, F=3,946, p<.05, \eta^{2}$ parcial $=0,477$ ) reveló que el efecto del factor tiempo era estadísticamente significativo. Las diferencias eran estadísticamente significativas para las respuestas pasivas $\left(F=8,752, p=.01, \eta^{2}\right.$ parcial $\left.=0,368\right)$ y asertivas $\left(F=7,549, p<.05, \eta^{2}\right.$ parcial $=$ 
0,335), con tamaños del efecto grandes. De este modo, mientras que las respuestas asertivas aumentaron tras la intervención, las pasivas disminuyeron y las agresivas se mantuvieron iguales.

$\overline{\text { Tabla 3. Medias y desviaciones típicas en las distintas puntuaciones del CABS en ambos momentos temporales }(N=16)}$

\begin{tabular}{|l|c|c|c|}
\hline & $\begin{array}{c}\text { Pasivas } \\
(\mathbf{M}, \boldsymbol{D} \boldsymbol{t})\end{array}$ & $\begin{array}{c}\text { Agresivas } \\
(\boldsymbol{M}, \boldsymbol{D} \boldsymbol{t})\end{array}$ & $\begin{array}{c}\text { Asertivas } \\
(\boldsymbol{M}, \boldsymbol{D} \boldsymbol{t})\end{array}$ \\
\hline Pre & $9,06(3,13)$ & $2,06(2,21)$ & $15,88(4,30)$ \\
\hline Post & $7,13(2,73)$ & $2,13(1,86)$ & $17,69(3,50)$ \\
\hline
\end{tabular}

Nota: las puntuaciones para las respuestas pasivas, agresivas y asertivas se refieren al número de veces que el sujeto señala ese tipo de respuestas (máximo 54 para las pasivas y agresivas y 27 para las asertivas).

Fuente: elaboración propia.

\section{- Satisfacción del curso por parte de los participantes}

Los participantes mostraron un elevado grado de satisfacción con los contenidos trabajados, así como con la relación establecida con la profesional que impartió el curso. Las medias alcanzadas en los ítems En general, ¿te ha gustado esta asignatura? $(3,94)$ y ¿Cómo ha sido tu relación con el profesor o profesora de esta asignatura? $(4,94)$ mostraron un elevado grado de satisfacción de los participantes con los contenidos trabajados y con la relación establecida con la profesora. Respecto a las preguntas de tipo abierto, destacaron como aspectos positivos las actividades grupales y las dramatizaciones. Asimismo, señalaron la importancia de aprender a gestionar bien las emociones, especialmente el enfado. No señalaron ningún aspecto negativo.

\section{Discusión}

El objetivo del presente estudio fue mejorar la inteligencia emocional y la conducta social de un grupo de personas con discapacidad intelectual integrantes del Programa Universitario para el Empleo de Jóvenes con Discapacidad Intelectual (UNINCLUV) de la UV a través del curso "Gestión Emocional y Habilidades Sociales". El objetivo principal del curso era fomentar en los participantes la adquisición de las habilidades sociales y emocionales necesarias para poder relacionarse con éxito y trabajar satisfactoriamente en equipo en los potenciales entornos laborales.

Los resultados de este estudio pusieron de manifiesto que, si bien los participantes manifestaron un mayor número de estrategias asertivas y un menor número de estrategias pasivas ante la solución de problemas interpersonales tras su participación en el curso, no se produjeron cambios en sus puntuaciones en las cinco dimensiones de la inteligencia emocional evaluadas (interpersonal, intrapersonal, estrés, adaptabilidad y estado de ánimo). Por consiguiente, se puede concluir que la intervención realizada en el curso fue útil para aumentar la competencia social del alumnado, pero no así su competencia emocional. Estos resultados no irían en la línea de lo encontrado en estudios previos que hallaron cambios tanto en los indicadores de inteligencia emocional como de conducta social (González et al., 2019). 
Ante estos resultados pueden proponerse distintas explicaciones. Por una parte, las actividades planteadas en el curso iban dirigidas a trabajar preferentemente las habilidades sociales asertivas, de hecho, algunas de las situaciones que se trabajaron en el aula para desarrollar la asertividad de los participantes eran similares a las que se planteaban en el CABS, por lo que posiblemente les resultó más fácil plasmarlas en el cuestionario. Además, estaban basadas en dramatizaciones, de modo que el alumnado trabajaba principalmente el componente conductual, hecho que seguramente los llevó a aprenderlas y recordarlas mejor. Por otra parte, consideramos que para poder desarrollar y afianzar las competencias evaluadas a través del EQ-i:YV se necesitaría de una intervención más intensiva y distribuida en el tiempo. En este sentido, cabe destacar que la intervención desarrollada por González et al. (2019) en población con discapacidad intelectual en contexto penitenciario se desarrolló a lo largo de 8 talleres de una semana (12 horas) de duración cada uno, frente a las 16 horas repartidas en solo 4 sesiones del presente curso. La escasa duración de la intervención implementada se encontraría por tanto entre una de las limitaciones a destacar del presente estudio. Asimismo, distribuir esta intervención en el tiempo también favorecería que el alumnado dispusiese de mayor número de oportunidades para practicar y transferir lo aprendido en el aula a su vida diaria. Cabe también la posibilidad de que los instrumentos de medida utilizados en el presente estudio no hayan sido suficientemente sensibles para evaluar los efectos de la intervención. Esto se ha observado principalmente en aquellos instrumentos que usan una escala de respuesta tipo Likert con menos de 5 opciones (Bisquerra y Pérez-Escoda, 2015), como es el caso del EQ-i:YV. Futuros estudios deberían incorporar, en la medida de lo posible, medidas con una mayor sensibilidad para detectar cambios, como son aquellas con escalas de respuesta de 0 a 10 puntos. Otra limitación importante y ya señalada, sería la falta de un grupo control para poder conocer los efectos reales de la intervención en los participantes y eliminar el posible impacto de otros factores. Sin embargo, cabe señalar la dificultad para encontrar un grupo control entre la población de estas características dada la enorme heterogeneidad existente dentro del grupo de participantes y de la propia discapacidad intelectual (p. ej., edad, presencia de diagnósticos comórbidos, etc.). Por último, entre otras de las limitaciones cabría destacar el uso únicamente del autoinforme para conocer los posibles efectos de la intervención. Podrían haberse completado con la información obtenida de otros informantes (p. ej., la familia) o con registros de observación.

A pesar del carácter limitado de los resultados y de su poco alcance o generalización a otros participantes y contextos, cabe subrayar que se trata de un trabajo novedoso que aborda la evaluación de una intervención en educación emocional dentro de un programa de formación universitario en España. Los resultados de este estudio apuntan a la importancia de desarrollar este tipo de iniciativas en entornos universitarios. En este sentido, destacar el elevado nivel de satisfacción manifestado por el alumnado con su participación en el programa, así como las valoraciones cualitativas que hacían referencia, por una parte, a una mayor facilidad para hablar de sus propias emociones y regularlas, y por otra parte, a un mayor autoconocimiento y aceptación. El desarrollo de las competencias socioemocionales es necesario para favorecer la participación, integración e inclusión social de las personas con discapacidad intelectual. Y esto ha de hacerse en un contexto de socialización natural como es la universidad. La universidad puede y debe jugar un papel fundamental en la inclusión y desarrollo pleno de todos los individuos, con o sin discapacidad. 


\section{Referencias bibliográficas}

Aierdi, A. et al. (2008). Inteligencia Emocional. Educación Secundaria Obligatoria (14-16 años), San Sebastián: Diputación Foral de Guipúzcoa.

Bar-On, R. y Parker, J. D. A. (2000). Emotional Quotient Inventory: Youth Version (EQ-i:YV). Technical manual. Toronto: Multi-Health Systems.

Bisquerra, R. (2000). Educación emocional y bienestar. Barcelona: Praxis.

Bisquerra, R. (2002). “La competencia emocional”. En M. Álvarez y R. Bisquerra (coords.), Manual de orientación y tutoría (pp. 144/69-144/83). Barcelona: Praxis.

Bisquerra, R. et al. (2015). Inteligencia emocional en educación. Madrid: Síntesis.

Bisquerra, R. y Pérez, N. (2007). “Las competencias emocionales”. Educación XXI, 10, pp. 61-82.

Bisquerra, R. y Pérez-Escoda, N. (2015). “¿Pueden las escalas Likert aumentar en sensibilidad?”. REIRE, Revista d'Innovació i Recerca en Educació, 8(2), pp. 129-147.

Cerrillo, R. et al. (2013). "Inclusión de jóvenes con discapacidad intelectual en la Universidad". Revista de Investigación en Educación, 11(1), pp. 41-57.

Dunn, L. M. et al. (2007). PEABODY. Test de Vocabulario en Imágenes. Madrid: TEA Ediciones.

Fernández-Berrocal, P. et al. (2017). "Avances en la investigación sobre competencias emocionales en educación”. Revista Interuniversitaria de Formación del Profesorado, 88(31), pp.15-26.

Ferrándiz, C. et al. (2012). "Social and emotional intelligence in childhood and adolescence: Spanish validation of a measurement instrument". Revista de Psicodidáctica, 17(2), pp. 309-339.

Filella, G. et al. (2014). "Resultados de la aplicación de un programa de educación emocional en Educación Primaria". Estudios sobre Educación, 26, pp. 125-147.

Fuller, M. et al. (2006). "Listening to students: the experiences of disabled students of learning at university". En M. Adams y S. Brown (eds.), Towards Inclusive Learning in Higher Education: Developing Curricula for Disabled Students (pp. 32-43). Londres: Routledge Falmer.

Fundación Universia (2017). Universidad y Discapacidad. III Estudio sobre el grado de inclusión del sistema universitario español respecto de la realidad de la discapacidad. Recuperado de https://bit.ly/2JGxRyn.

González, G. et al. (2019). "Inteligencia emocional en personas reclusas con discapacidad intelectual”. Siglo Cero, 50(1), pp. 7-25.

IBM Corp Released (2013). IBM SPSS Statistics for Windows, Version 22.0. Armonk, NY: IBM Corp.

Izuzquiza, D. (2012). "El valor de la inclusión educativa de jóvenes con discapacidad intelectual en las instituciones de educación superior: El Programa Promentor”. Bordón. Revista de Pedagogía, 64(1), pp.109-125.

La Malfa, G. et al. (2009). "Emotional development and adaptive abilities in adults with intellectual disability: a correlation study between the Scheme of Appraisal of Emotional Development (SAED) and Vineland Adaptive Behavior Scale (VABS)". Research in Developmental Disabilities, 30(6), pp.1406-1412. 
Miller, K. D. et al. (2016). "Inclusive higher education is reaping benefits for individuals with intellectual disabilities: One program's story”. Think College Insight Brief, 29, pp.1-6.

Moore, E. J. y Schelling, A. (2015). "Postsecondary inclusion for individuals with an intellectual disability and its effects on employment". Journal of Intellectual Disabilities, 19(2), pp. 130-148.

Muñoz de Morales, M. y Bisquerra, R. (2013). “Diseño, aplicación y evaluación de un plan de educación emocional en Guipúzcoa: análisis cuantitativo”. EduPsykhé Revista de Psicología y Educación, 12(1), pp.3-22.

Navas, P. et al. (2010). "La conducta adaptativa en personas con discapacidad intelectual”. Siglo Cero: Revista Española sobre Discapacidad Intelectual, 41(235), pp. 28-48.

Palazón, A. et al. (2011). "Relación entre la aplicación de metodologías docentes activas y el aprendizaje del estudiante universitario". Bordón. Revista de Pedagogía, 63(2), pp. 27-41.

de la Peña, V. et al. (2003). "Comportamiento asertivo y adaptación social: Adaptación de una escala de comportamiento asertivo (CABS) para escolares de enseñanza primaria (6-12 años)". Revista Electrónica de Metodología Aplicada, 8(2), pp. 11-25.

Prohn, S. M. et al. (2019). "Supports' Perspectives on the Social Experiences of College Students with Intellectual Disability”. Inclusion, 7(2), pp. 111-124.

Rey, L. et al. (2013). "Subjective Quality of Life of People with Intellectual Disabilities: The Role of Emotional Competence on Their Subjective Well-Being". Journal of Applied Research in Intellectual Disabilities, 26(2), pp. 146-156.

Ross, J. et al. (2013). "Postsecondary education employment and independent living outcomes of persons with autism and intellectual disability". Journal of Postsecondary Education and Disability, 26(4), pp. 337-351.

Ryan, J. y Struths, J. (2004). "University Education for All? Barriers to Full Inclusion of Students with Disabilities in Australian Universities". International Journal of Inclusive Education, 8(1), pp. 73-90.

Verdugo, M. A. et al. (2011). Discapacidad intelectual: definición, clasificación y sistemas de apoyo. Undécima edición. Madrid: Alianza Editorial.

Woods, R. et al. (1978). Assessment of assertive behavior in elementary school children. Chicago: Association for Advancement of Behavior Therapy. 